\title{
Miuraenamides A and B, Novel Antimicrobial Cyclic Depsipeptides from a New Slightly Halophilic Myxobacterium: Taxonomy, Production, and Biological Properties
}

\author{
Takashi Iizuka, Ryosuke Fudou, Yasuko Jojima, Sumie Ogawa, Shigeru Yamanaka, \\ Yasutaka Inukai, Makoto Ojika
}

Received: May 16, 2006 / Accepted: July 5, 2006

(C) Japan Antibiotics Research Association

\begin{abstract}
A slightly halophilic myxobacterial strain, $\mathrm{SMH}-27-4$, was isolated from nearshore soil and shown to belong to a new myxobacterium genus based on phylogenetic analysis. This slowly-growing myxobacterium produced the novel antibiotic depsipeptides miuraenamides A and B. Their physico-chemical properties and molecular formulas, $\mathrm{C}_{34} \mathrm{H}_{42} \mathrm{~N}_{3} \mathrm{O}_{7} \mathrm{Br}$ and $\mathrm{C}_{34} \mathrm{H}_{42} \mathrm{~N}_{3} \mathrm{O}_{7} \mathrm{I}$, were determined. Miuraenamides A exhibited potent and selective inhibition against a phytopathogenic microorganism, Phytophthora sp., and moderate inhibition against some fungi and yeasts, but was ineffective against bacteria. Both of the metabolites inhibited NADH oxidase at $\mathrm{IC}_{50}$ values of $50 \mu \mathrm{M}$, suggesting, like $\beta$-methoxyacrylate-type antibiotics, the electron transfer system of the mitochondrial respiratory chain as the cellular target.
\end{abstract}

Keywords myxobacterium, antibiotics, depsipeptides, polyketide, electron transport inhibitor

\section{Introduction}

Myxobacteria are considered a rich source of unique antibiotics that are rarely produced by other bacteria, actinomycetes and fungi [1 4]. Although myxobacterial

R. Fudou (Corresponding author), T. Iizuka, Y. Jojima: Microbiology Group, Institute of Life Sciences, Ajinomoto Co., Inc., 1-1 Suzuki-cho, Kawasaki-ku, Kawasaki 210-8681, Japan, E-mail: ryosuke_fudou@ajinomoto.com

S. Ogawa, S. Yamanaka: Faculty of Textile Science and Technology, Shinshu University, 3-15-1 Tsuneda, Ueda 386-8567, strains are rather difficult to isolate and to culture, the potential usefulness of their novel secondary metabolites has been frequently mentioned [4]. Myxobacteria are not only soil bacteria, but inhabit a wide range of natural environments. For example, truely halophilic myxobacteria were isolated from the marine environment in Japan [5]. Moreover, new antifungal compounds, such as haliangicins are produced by a marine myxobacterial strain SMP-2 which belongs to the new genus Haliangium [6 8]. These findings suggested novel myxobacteria as promising sources of new compounds. Therefore, we have continued the effort to isolate unknown myxobacteria from the coastal

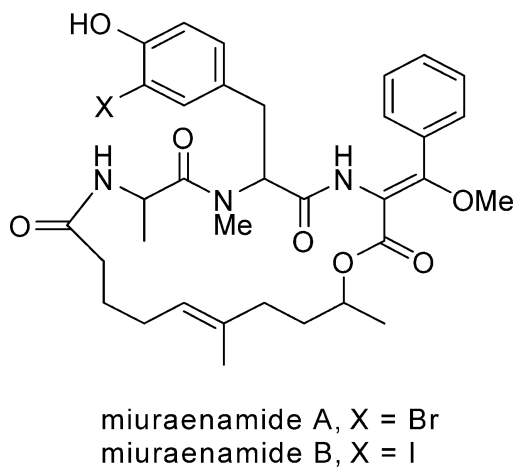

Fig. 1 Structures of miuraenamides.
Japan

Y. Inukai, M. Ojika: Graduate School of Bioagricultural Sciences, Nagoya University, Furo-cho, Chikusa-ku, Nagoya 4648601, Japan

* AJ-: No. of the Ajinomoto Culture Collection. 
samples. In the course of our search for myxobacterial antibiotics [9 12], miuraenamides A and B (Fig. 1) were obtained from the fermentation broth of a new slightly halophilic myxobacterium isolated from a nearshore soil in Miura Peninsula, Japan. We now describe the taxonomy and fermentation characteristics of this myxobacterium as well as the isolation, physicochemical properties and biological activity of these antibiotics.

\section{Materials and Methods}

\section{Microorganisms}

The myxobacterial strain SMH-27-4 (=AJ-110262*) was isolated from a soil sample collected in a brush vegetation near the seashore of Arai-Hama beach in the Miura Peninsula, Kanagawa, Japan. The principal method used for isolation of myxobacteria has been described previously, except that low salt concentration media (one fifth the strength of SWS solution, or $1 / 5 \mathrm{SWS}$ ) were used [5]. Phylogenetic and physiological studies were conducted as described [5].

\section{Fermentation}

The myxobacterial strain was cultivated at $27^{\circ} \mathrm{C}$ in Vy2$1 / 5 \mathrm{SWS}$ medium. The Vy2-1/5SWS medium contained (amount/liter) Baker's yeast cake (5 g, Wako Pure Chemical, Osaka, Japan), $\mathrm{NaCl}(10 \mathrm{~g})$, cyanocobalamin $(0.5 \mathrm{mg})$, SWS solution [5] $(200 \mathrm{ml})$ and agar $(15 \mathrm{~g})$, at $\mathrm{pH} 7.2$ adjusted with $\mathrm{NaOH}$. SWS is composed of the elements of seawater, such as magnesium, potassium, calcium, sulfate, carbonate, boric acid, strontium, iron, manganese and iodine [5]. The V-1/5SWS-Br medium was used for the production of antibiotics. The medium contained (per liter of 1/5 SWS) Baker's yeast cake (8g, Wako Pure Chemical), Yeast Extract (0.1 g, Difco Lab., Becton Dickinson \& Co., Sparks, MD, USA), NaCl (1.0 g), NaBr (7.0 g), HEPES $(2.0 \mathrm{~g})$ and the adsorber resin SP207 (20 g, Mitsubishi Chemical Co., Tokyo, Japan), at pH 7.3 adjusted with $\mathrm{NaOH}$. Fermentation was conducted on a rotary shaker (180 rpm) at $27^{\circ} \mathrm{C}$.

\section{Chromatography and Physicochemical Properties}

Flash chromatography was conducted using a low-pressure gradient system equipped with an 880-PU HPLC pump and an 880-02 gradient unit (Jasco, Tokyo, Japan). Preparative HPLC was performed on a high-pressure gradient system equipped with PU-1586 and PU-2086 pumps and a UV-1570 detector (Jasco). Specific rotations were determined using a DIP-370 digital polarimeter (Jasco). FT-IR spectra were recorded on a FT-IR-7000S spectrometer (Jasco).
UV spectra were recorded on an Ubest-50 UV/VIS spectrophotometer (Jasco). Mass spectra (MS) were recorded on a Mariner Biospectrometry Workstation (Applied Biosystems, Foster City, CA, USA) in the positive ESI mode. A peptide mixture containing angiotensin I, bradykinin and neurotensin was used as an internal standard for high-resolution MS analysis. NMR spectra were recorded on an AMX2 $600(600 \mathrm{MHz})$ spectrometer (Bruker). The NMR chemical shifts for ${ }^{1} \mathrm{H}$ and ${ }^{13} \mathrm{C}$, respectively, were referenced to the solvent peaks of $\delta_{\mathrm{H}}$ 7.26 (residual $\mathrm{CHCl}_{3}$ ) and $\delta_{\mathrm{C}} 77.0 \mathrm{ppm}$ for $\mathrm{CDCl}_{3}$ solutions.

\section{Isolation of Miuraenamides A and B}

The bacterial cells and the adsorber resin were separated from 14 liters of culture broth by centrifugation followed by two extractions with acetone (1.4 liters) at room temperature for two days. The extracts were combined and concentrated to give an aqueous mixture, which was extracted three times with EtOAc $(50 \mathrm{ml})$. After concentration, the residue (495 mg) was subjected to flash chromatography on silica gel [HI-FLASH SI-40W-M $(12 \mathrm{~g}, 20 \times 60 \mathrm{~mm}$, Yamazen, Osaka), 5 50\% (90 minutes), 50 100\% (50 minutes) EtOAc in $\mathrm{CHCl}_{3}, 4 \mathrm{ml} /$ minute]. The fractions, eluted with $10 \sim 30 \%$ EtOAc in $\mathrm{CHCl}_{3}$, were combined and concentrated to produce an oil $(69 \mathrm{mg})$, which was subjected to chromatography on silica gel [Wakogel C-300 $(7 \mathrm{~g}, 12 \times 130 \mathrm{~mm}$, Wako Pure Chemical), hexane - EtOAc $(1: 1,1: 2,1: 3$, and $1: 4)]$. The fractions eluted with hexane-EtOAc $(1: 3$ and $1: 4)$ were combined and evaporated. The residue $(17.3 \mathrm{mg})$ was subjected to reversed-phase HPLC [Develosil ODS UG-5 (10 i.d. $\times$ $250 \mathrm{~mm}$, Nomura Chemical, Aichi, Japan), 70\% MeOH, $8 \mathrm{ml} /$ minute, detection at $260 \mathrm{~nm}$ ] to yield miuraenamides A (10.9 mg, $\mathrm{Rt}=31.6$ minutes $)$ and $\mathrm{B}(0.5 \mathrm{mg}, \mathrm{Rt}=35.6$ minutes).

\section{Antimicrobial Properties}

Anti-Phytophthora (a fungus-like phytopathogen) activity of the extracts of culture broths and chromatographic fractions was assessed according to the method of Ojika et al. [9]. The antimicrobial spectrum was obtained using the serial liquid media dilution method using Yeast Nitrogen Base broth (Difco) with $0.5 \%$ glucose for yeast and filamentous fungi, and Mueller-Hinton broth (Difco) for bacteria. Approximately $10^{4}$ freshly grown cells (or spores) of the test strains were inoculated and grown at $25^{\circ} \mathrm{C}$ for yeast and fungi and at $37^{\circ} \mathrm{C}$ for bacteria. For the non spore-forming fungal species (Phytophthora capsici and Trichophyton mentagrophytes), small pieces of agar (approximately $1 \mathrm{~mm}^{3}$ ) containing the mycelia were used as 
inocula. After incubation for 72 hours for filamentous fungi and 24 hours for yeasts and bacteria, the minimal inhibitory concentrations (MICs) were determined.

\section{NADH Oxidase Inhibition}

The inhibition rate of fungal NADH oxidase in Candida rugosa AJ14513 mitochondrial particles was determined using a slight modification of Thierbach et al. [13]. The fungus was cultured with shaking in YM medium at $25^{\circ} \mathrm{C}$ for 40 hours and used for further experiments. A mitochondrial fraction of the fungal cells was extracted according to the method described by Fang et al. [14].

\section{Results and Discussion}

\section{Taxonomic Studies}

The antibiotic depsipeptide producing strain SMH-27-4 (=AJ-110262) exhibited enlarging colony formation on agar plates and conspicuous bacteriolytic action; both features are typical characteristics of myxobacteria [4]. However, the strain did not form fruiting bodies whose shapes and structures are important taxonomic features of myxobacteria. Consequently, the strain was subjected to phylogenetic analysis based on 16SrDNA sequences of nearly $1500 \mathrm{bp}$ [DDBJ (=DNA Data Bank of Japan,

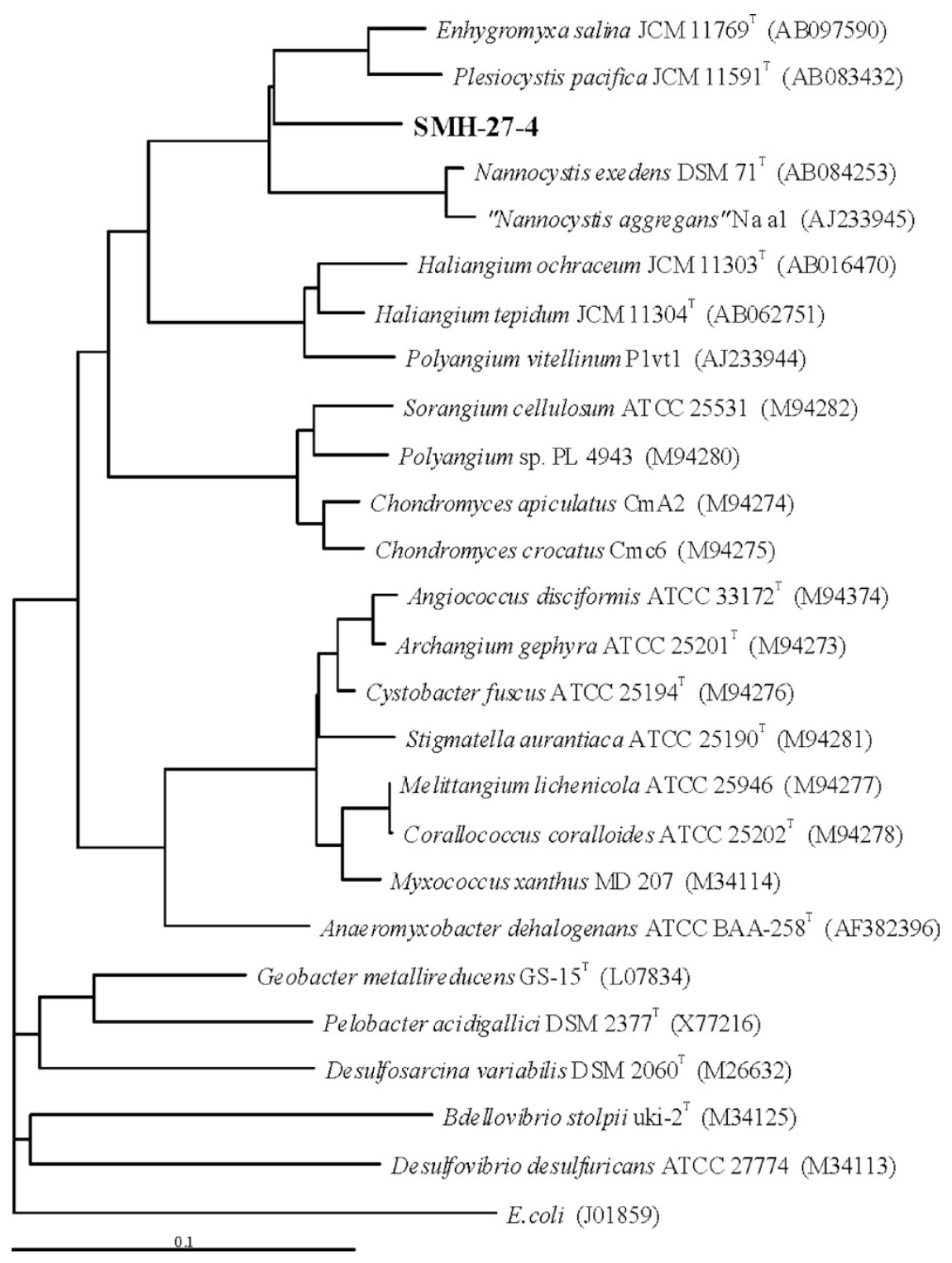

Fig. 2 Phylogenetic tree of the novel myxobacterial strain SMH-27-4 and other myxobacteria.

Bar, 0.1 changes per sequence position. 
National Institute of Genetics, Mishima, Shizuoka, Japan) Accession No. for the strain: AB252740]. The strain was shown to be related to the suborder Sorangineae myxobacteria, and was positioned near the genus Nannocystis and the marine myxobacterial genera Enhygromyxa and Plesiocystis [15, 16] (Fig. 2). Homology with its closest relatives was relatively low at $93.0 \%$ with Nannocystis exedens, 93.2 to $93.3 \%$ with Enhygromyxa salina, and 91.3 to $91.5 \%$ with Plesiocystis pacifica. The strain showed the slight salt requirement with an optimum $\mathrm{NaCl}$ concentration for growth of approximately 0.5 to $1.0 \%(\mathrm{w} / \mathrm{v})$, which is slightly lower than that observed for any myxobacteria of marine origin reported to date $[5,15$, 16]. Since these results strongly suggested that the strain might represent a novel taxon at the level of genus, we tentatively named it Paraliomyxa miuraensis. A more detailed description of the strain will be reported elsewhere.

\section{Production and Isolation of Antibiotics}

After the incubation of the myxobacterial strain on the Vy2-1/5SWS agar plates for 7 to 10 days, agar pieces, taken from the periphery of the colonies with a sterile spatula, were inoculated into 2-liter Erlenmeyer flasks containing $500 \mathrm{ml}$ of the production medium. Cultivation

Table 1 Physico-chemical properties of miuraenamides

\begin{tabular}{|c|c|c|}
\hline & Miuraenamide A & Miuraenamide B \\
\hline Appearance & White powder & White powder \\
\hline Molecular formula & $\mathrm{C}_{34} \mathrm{H}_{42} \mathrm{~N}_{3} \mathrm{O}_{7} \mathrm{Br}$ & $\mathrm{C}_{34} \mathrm{H}_{42} \mathrm{~N}_{3} \mathrm{O}_{7} \mathrm{I}$ \\
\hline ESI-MS & 684.2 and $686.2(49,60)[\mathrm{M}+\mathrm{H}]^{+}$ & $732.2(100)[\mathrm{M}+\mathrm{H}]^{+}$ \\
\hline$m / z$ (rel. int) & 706.2 and $708.2(96,100)[\mathrm{M}+\mathrm{Na}]^{+}$ & $754.2(24)[\mathrm{M}+\mathrm{Na}]^{+}$ \\
\hline HR MS found & $684.2278[\mathrm{M}+\mathrm{H}]$ & $732.2139[\mathrm{M}+\mathrm{H}]$ \\
\hline calcd & $684.2279\left(\mathrm{C}_{34} \mathrm{H}_{43} \mathrm{~N}_{3} \mathrm{O}_{7}{ }^{79} \mathrm{Br}\right)$ & $\left.732.2140\left(\mathrm{C}_{34} \mathrm{H}_{43} \mathrm{~N}_{3} \mathrm{O}_{7}\right)\right)$ \\
\hline$[\alpha]_{D}^{25}$ & +59 (с 0.15, MeOH) & +30 (c 0.02, MeOH) \\
\hline$U V \lambda_{\max }^{\mathrm{MeOH}} \mathrm{nm}(\varepsilon)$ & $203(49,000), 276(11,000)$ & $203(53,000), 274(11,000)$ \\
\hline $\mathrm{IR} v_{\max }\left(\right.$ film) $\mathrm{cm}^{-1}$ & $3336,1684,1654,1636,1509,1256,1217,1119,756$ & $3336,1683,1636,1507,1261,1217,1119,756$ \\
\hline HPLC Rt $t^{a}$ & 16.8 minutes & 19.0 minutes \\
\hline
\end{tabular}

${ }^{a}$ Develosil ODS UG-5 (4.6×250 mm), 70\% MeOH, $1.0 \mathrm{ml} /$ minute, UV $260 \mathrm{~nm}$.

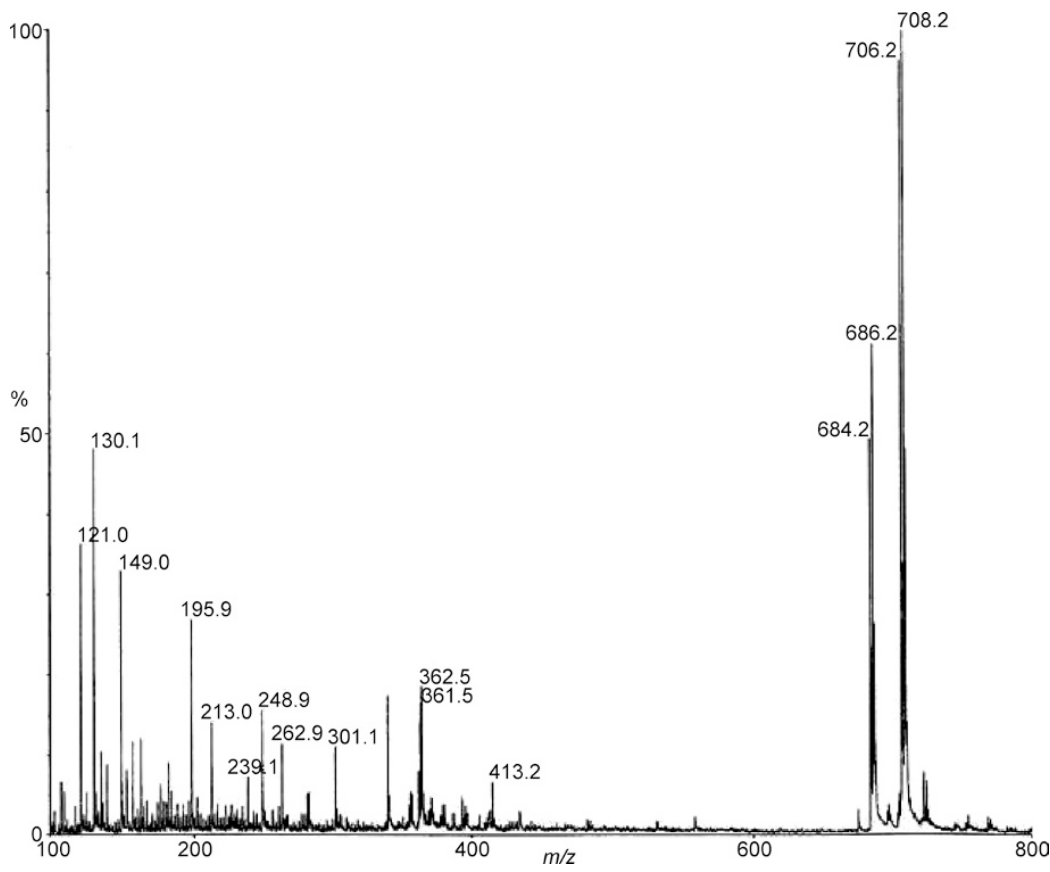

Fig. 3 ESI-TOF-MS(+) spectrum of miuraenamide A. 
was performed on a rotary shaker at $27^{\circ} \mathrm{C}$ for 18 days. A total of 14 liters of culture broth was used for further extraction and isolation. The bacterial cells, including the adsorber resin, were extracted with acetone then separated into the EtOAc and water fractions by solvent partition. The EtOAc fraction was chromatographed twice on silica gel before being purified by reversed-phase HPLC to give miuraenamides A (10.9 mg) and B (0.5 mg).

\section{Physicochemical Properties}

Table 1 summarizes some properties of miuraenamides A and B. The electrospray-ionization time-of-flight (ESI-

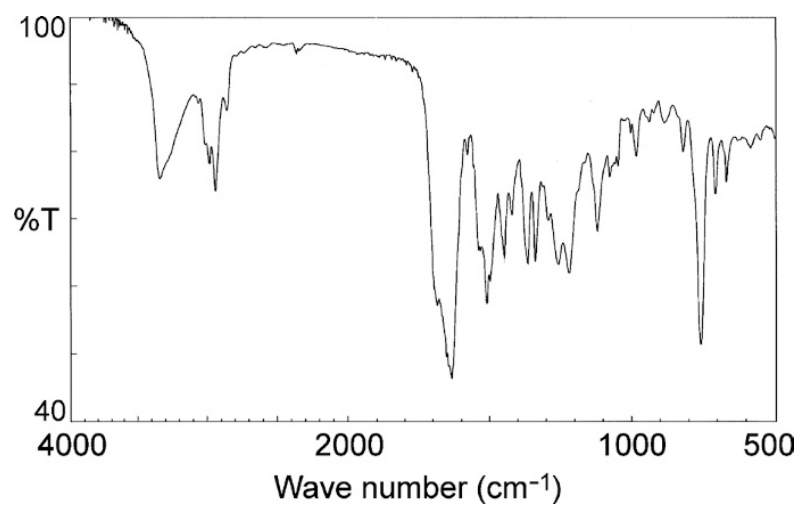

Fig. 4 IR spectrum of miuraenamide A (film).
TOF) MS of miuraenamide A showed protonated molecular ions at $m / z \quad 684.2$ and $686.2(\mathrm{M}+\mathrm{H})^{+}$at a ratio of approximately $1: 1$ (Fig. 3), indicating the presence of one bromine atom in the molecules. The molecular formulas were determined by high-resolution ESI-TOF MS. The IR bands of miuraenamide A at $1684 \mathrm{~cm}^{-1}$ (Fig. 4) and UV absorption at $276 \mathrm{~nm}$ (Fig. 5) suggest the presence of an unsaturated carbonyl group. The amide bands at 1654, 1636 , and $1509 \mathrm{~cm}^{-1}$, as well as three nitrogen atoms in the molecules, indicate the peptide nature of the molecules. The properties of miuraenamide B were relatively similar

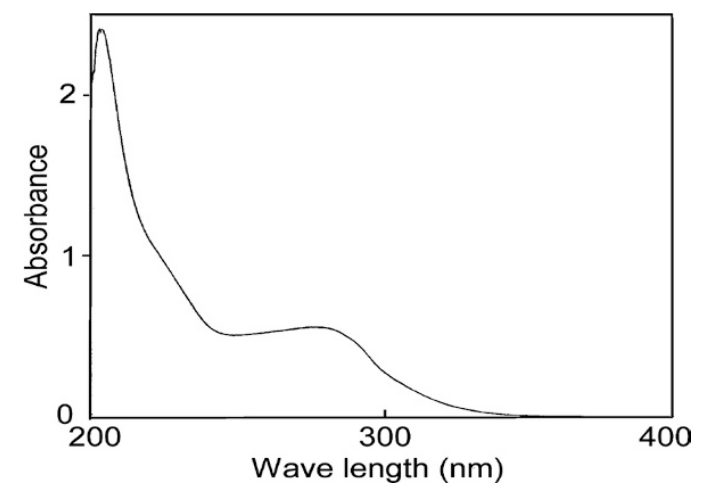

Fig. 5 UV spectrum of miuraenamide $A(\mathrm{MeOH}$, $\left.\mathrm{c}=5.1 \times 10^{-5} \mathrm{M}\right)$.

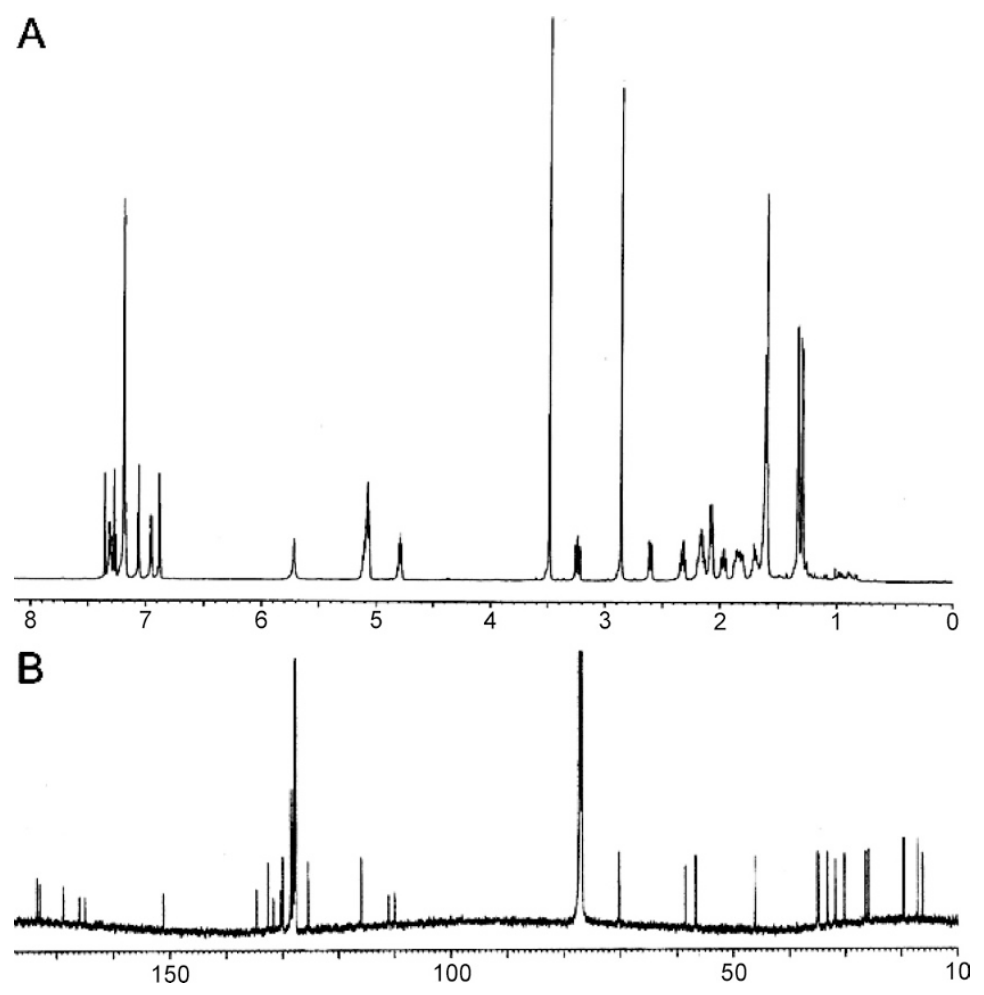

Fig. $6{ }^{1} \mathrm{H}(\mathrm{A})$ and ${ }^{13} \mathrm{C}(\mathrm{B}) \mathrm{NMR}$ spectra of miuraenamide $\mathrm{A}$ in $\mathrm{CDCl}_{3}\left[600 \mathrm{MHz}\right.$ for $\left.{ }^{1} \mathrm{H}\right]$. 


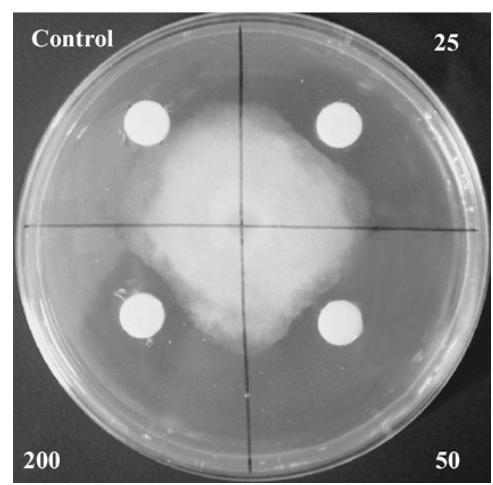

Fig. 7 Inhibition of $P$. capsici by miuraenamide A. [Doses: ng/disk].

Table 2 Antimicrobial spectrum of miuraenamide A

\begin{tabular}{lc}
\hline \multicolumn{1}{c}{ Tested microorganisms } & MIC $(\mu \mathrm{g} / \mathrm{ml})$ \\
\hline Fungi & \\
Absidia spinosa AJ 117196 & 12.5 \\
Aspergillus niger AJ 117065 & $>50$ \\
Botrytis cinerea AJ 117140 & $>50$ \\
Phytophthora capsici NBRC 8386 & 0.4 \\
Rhizopus oryzae AJ 117321 & 6.3 \\
Trichophyton mentagrophytes AJ 11716 & 12.5 \\
Yeast & 12.5 \\
Candida rugosa AJ 14513 & $>50$ \\
Saccharomyces cerevisiae AJ 14587 & 25 \\
Pichia burtonii AJ 14071 & 12.5 \\
Rhodotorula minuta AJ 5017 & \\
Bacteria & $>50$ \\
Escherichia coli NIHJ & $>50$ \\
Staphylococcus aureus AJ 12510 & $>50$ \\
Bacillus subtilis ATCC 6051 & \\
\hline
\end{tabular}

to those of miuraenamide A except for the molecular formula, in which the bromine atom was replaced by iodine (Fig. 1). The ${ }^{1} \mathrm{H}$ and ${ }^{13} \mathrm{C}$ NMR spectra of miuraenamide $\mathrm{A}$ are shown in Fig. 6. The details of the chemical attributes of the molecules such as elucidation of the structure will be published elsewhere.

\section{Biological Activity}

Miuraenamides A and B were obtained by antiPhytophthora screening using a paper disk diffusion method. Both compounds inhibited the phytopathogenic oomycete, Phytophthora capsici, at a minimum dose of $25 \mathrm{ng} /$ disk (Fig. 7). Table 2 shows the antimicrobial spectrum of miuraenamide A. We could not determine the spectrum of miuraenamide $B$ due to the scantiness of the

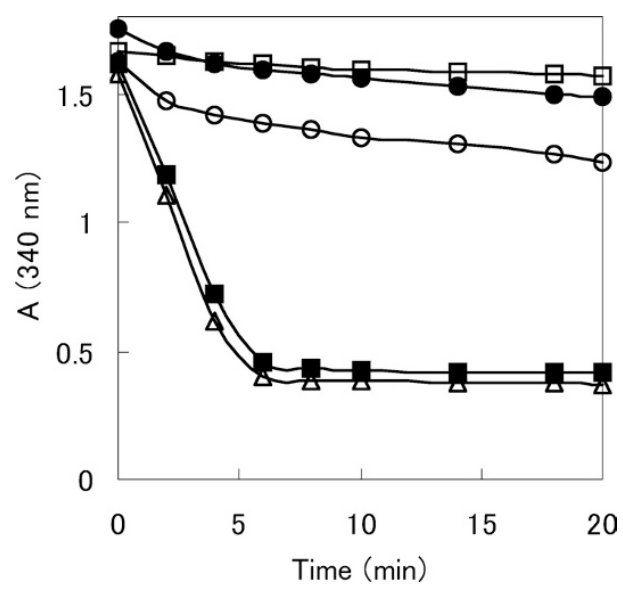

Fig. 8 Time-dependent inhibition of NADH oxidase by miuraenamides and other antibiotics.

O: miuraenamide $\mathrm{A}(100 \mu \mathrm{M})$; 0 : miuraenamide $\mathrm{B}(100 \mu \mathrm{M})$; $\square$ : cystothiazole $\mathrm{A}(10 \mu \mathrm{M}) ; \mathbf{\square}$ : amphotericin $\mathrm{B}(10 \mu \mathrm{M}) ; \triangle$ : control $(\mathrm{MeOH})$

compound available. The spectrum may be very similar to that of miuraenamide A, because of the structural similarity (Fig. 1) and the same anti-Phytophthora activity. The compound selectively inhibited Phytophthora but did not inhibit bacteria. One of the interesting structural features is the $\beta$-methoxyacrylate (and enamide $\mathrm{NH}-\mathrm{C}=\mathrm{C}$ ) moiety of the dehydrophenylalanine residue. Since the $\beta$ methoxyacrylate-type metabolites are known to be antifungal respiratory-chain inhibitors, e.g., strobilurins [17], cystothiazoles [9] and haliangicins [8], the inhibition of NADH oxidase was evaluated using Candida-derived mitoplast (Fig. 8) and it was found that miuraenamides inhibited NADH oxidase activity at $100 \mu \mathrm{M}$. Cystothiazole A, which is a typical $\beta$-methoxyacrylate compound previously isolated from a myxobacterium by us [9], was used as a positive control and completely inhibited NADH oxidase at $10 \mu \mathrm{M}$. On the other hand, amphotericin B, which is a potent antifungal compound of the heptaenemacrolide, serving as negative control, did not show inhibition. The $\mathrm{IC}_{50}$ values of $50 \mu \mathrm{M}$ were determined in an experiment using various concentrations (data not shown). The results suggest the electron transfer system of the mitochondrial respiratory chain as cellular target, similar to $\beta$-methoxyacrylate compounds. The miuraenamides are the second reported example of $\alpha$-nitrogen-linked $\beta$ methoxyacrylates produced by bacteria since the discovery of cyrmenins [18, 19].

Acknowledgment The authors wish to thank Mr. Masaru Ishihara of Ajinomoto Co., for his excellent technical assistance. 


\section{References}

1. Reichenbach H, Höfle G. Production of Bioactive Secondary Metabolites. In Myxobacteria II. Eds., M. Dworkin and D. Kaiser, pp. 347-397, American Society for Microbiology, Washington DC (1993)

2. Reichenbach H, Gerth K, Irschik H, Kunze B, Höfle G. Myxobacteria: a source of new antibiotics. Trends Biotechnol 6: 115-121 (1988)

3. Reichenbach H, Höfle G. Myxobacteria as Producers of Secondary Metabolites. In Drug Discovery from Nature. Eds., S. Grabley and R. Thiericke, pp. 149-179, Springer, Berlin (1999)

4. Gerth K, Pradella S, Perlova O, Beyer S, Müller R. Myxobacteria: proficient producers of novel natural products with various biological activities - past and future biotechnological aspects with the focus on the genus Sorangium. J Biotechnol 106: 233-253 (2003)

5. Iizuka T, Jojima Y, Fudou R, Yamanaka S. Isolation of myxobacteria from the marine environment. FEMS Microbiol Lett 169: 317-322 (1998)

6. Fudou R, Iizuka T, Yamanaka S. Haliangicin, a novel antifungal metabolite produced by a marine myxobacterium. 1. Fermentation and biological characteristics. J Antibiot 54: 149-152 (2001)

7. Fudou R, Iizuka T, Sato S, Ando T, Shimba N, Yamanaka S. Haliangicin, a novel antifungal metabolite produced by a marine myxobacterium. 2. Isolation and structural elucidation. J Antibiot 54: 153-156 (2001)

8. Kundim BA, Itou Y, Sakagami Y, Fudou R, Iizuka T, Yamanaka S, Ojika M. New haliangicin isomers, potent antifungal metabolites produced by a marine myxobacterium. J Antibiot 56: 630-638 (2003)

9. Ojika M, Suzuki Y, Tsukamoto A, Sakagami Y, Fudou R, Yoshimura T, Yamanaka S. Cystothiazoles A and B, new bithiazole-type antibiotics from the myxobacterium Cystobacter fuscus. J Antibiot 51: 275-281 (1998)

10. Suzuki Y, Ojika M, Sakagami Y, Fudou R, Yamanaka S.
Cystothiazoles $\mathrm{C} \sim \mathrm{F}$, new bithiazole-type antibiotics from the myxobacterium Cystobacter fuscus. Tetrahedron 54: 11399-11404 (1998)

11. Akita H, Sasaki T, Kato K, Suzuki Y, Kondo K, Sakagami Y, Ojika M, Fudou R, Yamanaka S. (+)-Cystothiazole G: isolation and structural elucidation. Tetrahedron 60: 4735-4738 (2004)

12. Kundim BA, Itou Y, Sakagami Y, Fudou R, Yamanaka S, Ojika M. Novel antifungal polyene amides from the myxobacterium Cystobacter fuscus: isolation, antifungal activity and absolute structure determination. Tetrahedron 60: 10217-10221 (2004)

13. Thierbach G, Reichenbach H. Myxothiazol, a new inhibitor of the cytochrome b-c1 segment of the respiratory chain. Biochim Biophys Acta 638: 282-289 (1981)

14. Fang J, Beattie DS. External alternative NADH dehydrogenase of Saccharomyces cervisiae: a potential source of superoxide. Free Radical Biol Med 34: 478-488 (2003)

15. Iizuka T, Jojima Y, Fudou R, Hiraishi A, Ahn J-W, Yamanaka S. Plesiocystis pacifica gen. nov., sp. nov., a marine myxobacterium that contains dihydrogenated menaquinone, isolated from the Pacific coasts of Japan. Int. J. Syst. Evol. Microbiol. 53: 189-195 (2003)

16. Iizuka T, Jojima Y, Fudou R, Tokura M, Hiraishi A, Yamanaka S. Enhygromyxa salina gen. nov., sp. nov., a slightly halophilic myxobacterium isolated from the coastal areas of Japan. Syst. Appl. Microbiol. 26: 189-196 (2003)

17. Sauter H, Steglich W, Anke T. Strobilurins: evolution of a new class of active substances. Angew Chem Int Ed 38: 1328-1349 (1999)

18. Sasse F, Leibold T, Kunze B, Höfle G, Reichenbach H. Cyrmenins, new $\beta$-methoxyacrylate inhibitors of the electron transport. Production, isolation, physico-chemical and biological properties. J Antibiot 56: 827-831 (2003)

19. Leibold T, Sasse F, Reichenbach H, Höfle G. Cyrmenins, novel antifungal peptides containing a nitrogen-linked $\beta$ methoxyacrylate pharmacophore: Isolation and structural elucidation. Eur J Org Chem 431-435 (2004) 